\title{
Microscopic structure and dynamics of air/water interface by computer simulations - comparison with sum-frequency generation experiments $\dagger$
}

\author{
Yanting Wang, ${ }^{a}$ Nathan O. Hodas, ${ }^{* b c}$ Yousung Jung ${ }^{d}$ and R. A. Marcus ${ }^{b}$ \\ Received 2nd December 2010, Accepted 9th February 2011 \\ DOI: $10.1039 / \mathbf{c 0 c p 0 2 7 4 5 f}$
}

\begin{abstract}
The air/water interface was simulated and the mode amplitudes and their ratios of the effective nonlinear sum-frequency generation (SFG) susceptibilities ( $A_{\mathrm{eff}}$ 's) were calculated for the ssp, ppp, and sps polarization combinations and compared with experiments. By designating "surface-sensitive" free $\mathrm{OH}$ bonds on the water surface, many aspects of the SFG measurements were calculated and compared with those inferred from experiment. We calculate an average tilt angle close to the SFG observed value of 35 , an average surface density of free $\mathrm{OH}$ bonds close to the experimental value of about $2.8 \times 10^{18} \mathrm{~m}^{-2}$, computed ratios of $A_{\text {eff' }}$ s that are very similar to those from the SFG experiment, and their absolute values that are in reasonable agreement with experiment. A one-parameter model was used to calculate these properties. The method utilizes results available from independent IR and Raman experiments to obtain some of the needed quantities, rather than calculating them $a b$ initio. The present results provide microscopic information on water structure useful to applications such as in our recent theory of on-water heterogeneous catalysis.
\end{abstract}

\section{Introduction}

The hydrogen $(\mathrm{H})$ bonded structure of water at the air/water interface $^{1-5}$ is of fundamental interest because it determines the properties of aqueous interfaces and their reactivity. Certain atmospheric reactions, ${ }^{6}$ such as those involved in ozone depletion, are known to be catalyzed by the ice surface on cloud particles. Study of the air/water interface is also important for understanding more complicated organic/water interfaces ${ }^{5}$ that have recently begun to attract interest for its potential for rate acceleration ${ }^{7}$ of organic reactions and green chemistry in emulsions. For example, interest in catalytic effects of dangling OH's has also been extended to their role in catalysis of different organic reactions by metal oxides enriched in surface $\mathrm{OH}$ groups. ${ }^{8}$

The first molecular structure of water at the air/water interface using the surface-specific vibrational sum-frequency generation (SFG) technique was reported by Du et al. ${ }^{9}$ in $1993 .{ }^{1,10}$ In that

\footnotetext{
${ }^{a}$ Key Laboratory of Frontiers in Theoretical Physics, Institute of Theoretical Physics and Kavli Institute for Theoretical Physics China, Chinese Academy of Sciences,

55 Zhongguancun East Road, Beijing 100190, P. R. China

${ }^{b}$ Department of Chemistry, California Institute of Technology, Pasadena,CA 91125.E-mail: hodas@caltech.edu

${ }^{c}$ Department of Physics, California Institute of Technology, Pasadena, CA 91125

${ }^{d}$ Graduate School of Energy, Environment, Water, and Sustainability (WCU), Korea Advanced Institute of Science and Technology (KAIST), Daejeon 305-701, Korea

$\dagger$ This article is part of the special collection on Interfacial processes and mechanisms in celebration of John Albery's 75th birthday.
}

work, using the "titration" of dangling $\mathrm{OH}$ groups with methanol and hence the complete suppression of the peak of the free $\mathrm{OH}$ SFG signal, it was found that about $25 \%$ of surface water molecules have one dangling $\mathrm{OH}$ bond that is not $\mathrm{H}$-bonded to other water molecules. It is thereby free, and protrudes out of the water phase, confirming earlier predictions from computations. ${ }^{11}$ Roughly the same picture of the existence of the free $\mathrm{OH}$ bonds at the interface has since been consistently observed in SFG experiments by Richmond, ${ }^{5}$ Eisenthal, ${ }^{12}$ Allen, ${ }^{13}$ and Gan et al.,${ }^{14}$ and also in computer simulations. ${ }^{3,15-19}$ The structure of water at the hydrophobic organic/water interfaces was shown to be similar to that at the air/water interface., ${ }^{9,20,21}$ In the process of understanding the air/water interface, different interpretations were also made in terms of details of the air/water interface using both experiments and computations. ${ }^{1,22-24}$ Although previous work has made significant progress toward explaining the observed SFG spectra using theory and simulation, 5,16,25-27 a complete picture of how rapid motion averages out the SFG has not been presented. In the present article, we make a quantitative comparison between multiple experimental observables, going beyond the comparison of ratios. In addition, by utilizing independent experimental results, coupled with molecular dynamics simulation of the air/water interface, the present work has as a goal a better understanding of motional averaging in SFG. This study was prompted by the recent "on-water" catalysis experiments ${ }^{7}$ and our interpretation in terms of the role of dangling $\mathrm{OH}$ groups at the water-oil emulsion interface. ${ }^{28}$

The orientation of free $\mathrm{OH}$ bonds at the air/water interface has been deduced from several SFG experiments to be nearly 
perpendicular to the surface plane. ${ }^{9,14,29}$ An SFG interpretation ${ }^{29}$ assumed a step-like distribution for the tilt angles $\left(\theta_{\mathrm{OH}}\right)$ between the free $\mathrm{OH}$ bonds and the surface normal, suggesting them to be in the interval $0^{\circ} \leq \theta_{\mathrm{OH}} \leq 51^{\circ}$, yielding an average tilt angle $\left\langle\theta_{\mathrm{OH}}\right\rangle \approx 35^{\circ}$. A more recent SFG experiment ${ }^{14}$ yielded a similar average tilt angle, $\left\langle\theta_{\mathrm{OH}}\right\rangle \approx 30^{\circ}$, with a narrow distribution width of $\leq 15^{\circ}$, assuming a Gaussian distribution model. In contrast to these distributions and average orientations of free $\mathrm{OH}$ bonds, however, previous molecular dynamics simulations $^{3,15-18,30}$ with various water models consistently predicted widths of the orientational distribution much broader than those assumed in the previous interpretations of SFG experiments. ${ }^{1,9,14,29}$ We address this discrepancy by considering the nature of free $\mathrm{OH}$ bonds on the surface, and, in a procedure detailed below, selectively removing those that would likely not contribute to the SFG, allowing a direct comparison between our calculations and experimental measurements.

\section{Methods}

The theoretical treatment we use in this paper to calculate the effective SFG nonlinear susceptibilities originated from the one previously developed in the literature ${ }^{9,29}$ to extract the orientation information of interfacial molecules from SFG experiments. Previous reviews have provided details of the treatment. ${ }^{31,32}$ Here, we summarize the assumptions and key expressions, for completeness, and implement the expressions to obtain the forms that are suitable for numerical calculations of the mode amplitudes by explicit integrals, rather than the more simplified fast and slow approximations. ${ }^{29}$

The SFG signal is proportional to the square of the effective nonlinear susceptibility, $\chi_{\mathrm{eff}}^{(2)}(\omega)$, which is a sum of resonant, $\chi_{R, \text { eff }}^{(2)}(\omega)$, and nonresonant, $\chi_{N R, \text { eff }}^{(2)}(\omega)$, contributions: ${ }^{1,10}$

$$
\chi_{\mathrm{eff}}^{(2)}(\omega)=\chi_{\mathrm{NR}, \mathrm{eff}}^{(2)}+\chi_{R, \mathrm{eff}}^{(2)}=\chi_{\mathrm{NR}, \mathrm{eff}}^{(2)}+\sum_{q} \frac{A_{q, \mathrm{eff}}}{\omega-\omega_{q}+i \Gamma_{q}}
$$

where $\omega$ is the incident infrared frequency, $\omega_{q}$ is the $q$ th vibrational frequency, $A_{q \text {, eff }}$ is the mode amplitude, and $\Gamma_{q}$ is the damping constant for the $q$ th molecular vibrational mode. The sum-frequency spectra are obtained by scanning the IR frequencies that probe the vibrational normal modes, using the IR-visible SFG method. The susceptibility originates at the air/water interface, caused by the symmetry breaking at the boundary. The bulk of the fluid has inversion symmetry and so does not contribute to techniques generating second-order nonlinear polarization.

SFG is approximated to be dipolar in origin, and we neglect any quadrupolar contribution that could emanate from the bulk. ${ }^{9}$ In this paper we focus on the free $\mathrm{OH}$ stretching mode of water molecules at the air/water interface, $\omega_{q}=3698 \mathrm{~cm}^{-1}$. This stretching frequency corresponds to the water configuration where one $\mathrm{OH}$ bond is free while the other $\mathrm{OH}$ bond of the same parent water molecule is still $\mathrm{H}$-bonded to neighboring water molecules (so-called "single-donor" configuration). The spectra for the ssp, ppp, and sps combinations of polarization, labeled in the order of the sum frequency output, visible input, and infrared input fields, are obtained in SFG experiments.

The $A_{\text {eff }}$ 's in eqn (1) can be related to the intrinsic tensor components via the Fresnel factors, i.e. via the macroscopic local field corrections, $L_{i i},{ }^{31}$

$$
\begin{aligned}
A_{\mathrm{eff}}(s s p)= & L_{y y}\left(\omega_{s}\right) L_{y y}\left(\omega_{1}\right) L_{z z}\left(\omega_{2}\right) \sin \beta_{2} A_{y y z} \\
A_{\mathrm{eff}}(p p p)= & -L_{x x}\left(\omega_{s}\right) L_{x x}\left(\omega_{1}\right) L_{z z}\left(\omega_{2}\right) \cos \beta_{s} \cos \beta_{1} \sin \beta_{2} A_{y y z} \\
& -L_{x x}\left(\omega_{s}\right) L_{z z}\left(\omega_{1}\right) L_{x x}\left(\omega_{2}\right) \cos \beta_{s} \sin \beta_{1} \cos \beta_{2} A_{y z y} \\
& +L_{z z}\left(\omega_{s}\right) L_{x x}\left(\omega_{1}\right) L_{x x}\left(\omega_{2}\right) \sin \beta_{s} \cos \beta_{1} \cos \beta_{2} A_{z y y} \\
& +L_{z z}\left(\omega_{s}\right) L_{z z}\left(\omega_{1}\right) L_{z z}\left(\omega_{2}\right) \sin \beta_{s} \sin \beta_{1} \sin \beta_{2} A_{z z z} \\
A_{\mathrm{eff}}(s p s)= & L_{y y}\left(\omega_{s}\right) L_{z z}\left(\omega_{1}\right) L_{y y}\left(\omega_{2}\right) \sin \beta_{1} A_{y z y}
\end{aligned}
$$

where $\beta_{S}, \beta_{1}$, and $\beta_{2}$ are the reflected or incident angles of sum frequency output, visible input, and infrared input, respectively. For an azimuthally isotropic air/water interface and assuming the visible frequency $\left(\omega_{1}\right)$ and the sum frequency $\left(\omega_{S}\right)$ are far removed from the electronic resonances, there are only three independent nonvanishing components, $A_{x x z}=A_{y y z}$, $A_{x z x}=A_{y z y}=A_{z x x}=A_{z y y}$, and $A_{z z z} \cdot{ }^{31}$ The $\omega_{2}$ is the infrared input light source and the $L_{i i}$ 's are defined in terms of the frequency-dependent effective dielectric constant of the interfacial layer, $\varepsilon(\omega)$, an air/water interface in our case. For the air/water interface, $\varepsilon\left(\omega_{\mathrm{S}}\right)=\varepsilon\left(\omega_{1}\right)=1.31$ and $\varepsilon\left(\omega_{2}\right)=1.2$ were estimated $^{29}$ from a 3-layer model. The numerical values of all these variables can be found in Table 1 of ref. 29. The $A_{i j k}$ 's are intrinsic (macroscopic) properties of the interface, whereas the experimental observables, $A_{\text {eff }}$ 's, are affected by the frequencydependent dielectric response of the interface medium as described above and by the experimental conditions. Therefore, using eqn (2), we use the above $\varepsilon$ 's to obtain $A_{\text {eff }}$ 's from the calculated $A_{i j k}$ 's.

The $A_{i j k}$ 's in eqn (2) are the resonant components of macroscopic susceptibility tensor, and can be represented at a molecular level as an ensemble average of the molecular hyperpolarizability in the body fixed frame, $\beta_{q, \lambda \mu \nu}$ (corresponding to the hyperpolarizability $A_{q}$ of Du et al. ${ }^{9}$ ), of the interfacial water molecules in the lab frame (denoted by $i j k$ ), where $q$ corresponds to the IR resonant mode of the free $\mathrm{OH}$ bond. The derivation begins with the Fourier-Laplace transform of the classical SFG response function: related references are $26,29,33-36$

$$
\begin{aligned}
A_{i j k} \equiv & \left(\omega_{2}-\omega_{q}+i \Gamma_{q}\right) \chi_{R, i j k}^{(2)} \\
= & -\left(\omega_{2}-\omega_{q}+i \Gamma_{q}\right)\left(k_{\mathrm{B}} T\right)^{-1} \\
& \times \int_{0}^{\infty} e^{\mathrm{i} \omega_{2} t}\left\langle\alpha_{i j}(0) \mu_{k}(t)\right\rangle d t,
\end{aligned}
$$

Table 1 Average number of free $\mathrm{OH},\left\langle n_{\mathrm{OH}}\right\rangle$, and average orientation angle, $\left\langle\theta_{\mathrm{OH}}\right\rangle$, of free surface $\mathrm{OH}$ bonds calculated from three MD simulations with different snapshot time intervals

\begin{tabular}{lll}
\hline Snapshot interval & $\left\langle n_{\mathrm{OH}}\right\rangle$ & $\left\langle\theta_{\mathrm{OH}}\right\rangle$ \\
\hline $1 \mathrm{fs}$ & 26 & $38^{\circ}$ \\
$10 \mathrm{fs}$ & 26 & $38^{\circ}$ \\
$100 \mathrm{fs}$ & 26 & $38^{\circ}$ \\
\hline
\end{tabular}


where $\alpha_{i j}$ is the instantaneous polarizability tensor of the entire surface, and $\mu_{k}$ is the instantaneous dipole moment vector of the surface. Eqn (3) can be expanded as

$$
\begin{aligned}
A_{i j k}=- & \left(\omega_{2}-\omega_{q}+i \Gamma_{q}\right) \sum_{\lambda \mu \nu}\left(k_{\mathrm{B}} T\right)^{-1} \alpha_{\lambda \mu} \mu_{\nu} \\
& \times \int_{0}^{\infty} d t e^{i \omega_{2} t}\left\langle\sum_{n}^{N_{\mathrm{OH}}(0)} \sum_{m}^{N_{\mathrm{OH}}(t)} D_{i \lambda}^{n}(0) D_{j \mu}^{n}(0) D_{k \nu}^{m}(t)\right\rangle
\end{aligned}
$$

where the sum of $\lambda, \mu$, or $\nu$ is over $\hat{u}, \hat{v}$, or $\hat{w}$, the basis of the body-fixed molecular reference frame. Similarly, $i=\hat{x}, \hat{y}, \hat{z}$, the laboratory frame basis, and the same for $j$ and $k$. The sum includes the $n$th and $m$ th free surface $\mathrm{OH}$ bonds at times 0 and $t$, respectively. At time $t$ there are $N_{\mathrm{OH}}(t)$ free $\mathrm{OH}$ bonds. $D_{i \lambda}^{n}, D_{j \mu}^{n}$, and $D_{k \nu}^{m}$ are the time-dependent direction cosine matrix elements for the $n$th and $m$ th free surface $\mathrm{OH}$ bonds, defined as $D_{x u}=\hat{x} \cdot \hat{u}$. Eqn (4) is a generalization of equations in Wei and Shen, and it can be shown to reduce to corresponding approximations of slow and fast orientational dynamics. Given this, however, eqn (4) also explicitly incorporates spatial correlations between water molecules, unlike those in Wei and Shen, which use a mean-field approximation.

Comparison with the experimental results of Wei and Shen requires calculating the absolute value of the $A_{\text {eff }}$ 's. After substituting in the hyperpolarizability, we then calculate the quantities:

$$
\begin{aligned}
\operatorname{Re} A_{i j k}= & -\Gamma_{q} \sum_{\lambda \mu \nu} \beta_{q, \lambda \mu \nu} \int_{0}^{\infty} d t \cos \left(\omega_{q} t\right) \\
& \times\left\langle\sum_{n}^{N_{\mathrm{OH}}(0)} \sum_{m}^{N_{\mathrm{OH}}(t)} D_{i \lambda}^{n}(0) D_{j \mu}^{n}(0) D_{k \nu}^{m}(t)\right\rangle, \\
\operatorname{Im} A_{i j k}= & -\Gamma_{q} \sum_{\lambda \mu \nu} \beta_{q, \lambda \mu \nu} \int_{0}^{\infty} d t \sin \left(\omega_{q} t\right) \\
& \times\left\langle\sum_{n}^{N_{\mathrm{OH}}(0)} \sum_{m}^{N_{\mathrm{OH}}(t)} D_{i \lambda}^{n}(0) D_{j \mu}^{n}(0) D_{k \nu}^{m}(t)\right\rangle .
\end{aligned}
$$

We approximate the hyperpolarizability of $\mathrm{H}_{2} \mathrm{O}$ at $\omega_{2}=\omega_{q}=$ $3698 \mathrm{~cm}^{-1}$ by the "bond hyperpolarizability" of the free $\mathrm{OH}$ bond with cylindrical symmetry. This assumption simplifies eqn (5) by converting it to a form that can be evaluated without the knowledge of molecular hyperpolarizability of all individual water molecules in different environments. Under this assumption, $\beta_{q, \lambda \mu \nu}$ has only two nonvanishing components, $\beta_{\perp, \perp, \|}$ and $\beta_{\|,\|, \|}$, where $\|$and $\perp$ denote the body-fixed axes along and perpendicular to the free $\mathrm{OH}$ bond direction, respectively: $\beta_{\|} \equiv \beta_{\|,\|, \|}=\beta_{w w w}=2.88 \times 10^{-27} \mathrm{~m}^{4} \mathrm{~V}^{-1} \mathrm{~s}$ and $\beta_{\perp} \equiv \beta_{\perp, \perp, \|}=\beta_{u u w}=\beta_{v v w}=0.32 \beta_{\|}$, which were obtained from Raman and IR measurements. ${ }^{9}$ Therefore, the sum over $(\lambda, \mu, \nu)$ only has three non-vanishing combinations: $(\hat{u}, \hat{u}, \hat{w}),(\hat{v}, \hat{v}, \hat{w}),(\hat{w}, \hat{w}, \hat{w})$.

The time-dependent direction cosine matrix is $D_{l \xi}=\hat{l} \cdot \hat{\xi}(t)$, with $\hat{l}=(\hat{x}, \hat{y}, \hat{z})$ the fixed lab coordinates and $\hat{\xi}(t)=(\hat{u}, \hat{v}, \hat{w})$ the time-dependent molecular coordinates of the free $\mathrm{OH}$ bond. The water surface normal is along the $z$-axis of the laboratory coordinates, and the free $\mathrm{OH}$ bond forms an angle $\theta(t)$ with the surface normal at time $t$, where $\theta(t)$ lies in the interval $[0, \pi]$, and an angle $\phi(t)$ in the interval $[0,2 \pi]$ with respect to $\hat{x}$ in the $\hat{x}, \hat{y}$ plane. Then the expression of the molecular coordinates in the laboratory coordinates is

$$
\begin{aligned}
& \hat{u}=\cos \phi \cos \theta \hat{x}+\sin \phi \cos \theta \hat{y}-\sin \theta \hat{z} \\
& \hat{v}=-\sin \phi \cos \theta \hat{x}+\cos \phi \cos \theta \hat{y} \\
& \hat{w}=\cos \phi \sin \theta \hat{x}+\sin \phi \sin \theta \hat{y}+\cos \theta \hat{z}
\end{aligned}
$$

To evaluate the error introduced by finite simulation snapshot time interval, three MD simulations with different snapshot time intervals were used for the calculations, as described below.

In the MD simulations, 1264 water molecules were placed in a rectangular box of dimension $30 \times 30 \times 70 \AA^{3}$, where the water section is sandwiched between two sections of vapor along the $z$-direction. Periodic boundary conditions were used in all directions, but in the $z$-direction, we sandwiched the water slab with two slabs of vacuum. Water molecules were modeled by the DL_POLY $2 \operatorname{program}^{37}$ as rigid and nonpolarizable, using the TIP3P potential commonly used for molecular mechanics. ${ }^{38}$ The Nosé-Hoover thermostat ${ }^{39,40}$ was used to perform the constant NVT MD simulations at $T=298 \mathrm{~K}$ and the SHAKE algorithm ${ }^{41}$ was used to constrain the degrees of freedom of rigid water molecules. The time step for integrating Newton's equations is always $1 \mathrm{fs}$, but it is unnecessary to record the results of each step. Instead, we recorded the instantaneous orientation of all free $\mathrm{OH}$ bonds in discrete snapshots, evolving the simulation between snapshots without recording the individual steps. In this work, we saved all of the configurations for the snapshot time interval of $1 \mathrm{fs}$, and every 10 configurations for a time interval of $10 \mathrm{fs}$, and every 100 configurations for $100 \mathrm{fs}$. After adequate equilibration of a random initial configuration, simulated with a time step of $1 \mathrm{fs}$, three individual simulations each with a different snapshot time interval of either $1 \mathrm{fs}, 10 \mathrm{fs}$, or $100 \mathrm{fs}$ were performed. In the three cases, to generate a total of 40000 configurations, the total simulation time was $40 \mathrm{ps}, 400 \mathrm{ps}$, and $4 \mathrm{~ns}$, respectively. The correlation functions were calculated using a time average,

$$
\langle\alpha(0) \mu(t)\rangle=\frac{1}{T-t} \sum_{\delta t=0}^{T-t} \alpha(\delta t) \mu(t+\delta t),
$$

where $T$ is the length of the simulation and $\delta t$ is the offset time.

For sampled configurations of the water/vacuum interface, it is essential to select only the free $\mathrm{OH}$ bonds on the water surface that respond to the laser beams in real SFG experiments. Only the free $\mathrm{OH}$ bonds at the surface will break the inversion symmetry necessary for an SFG signal. Previous simulation studies ${ }^{3,15-18}$ identified surface free $\mathrm{OH}$ bonds as those positioned above a certain cutoff in the $z$-direction. However, as can be seen in Fig. 1, the water surface in the model fluctuates appreciably, and an arbitrary cutoff unavoidably includes the $\mathrm{OH}$ bonds in the bulk. Although isotropy due to random orientation of the free $\mathrm{OH}$ bond in the bulk will provide a natural surface selection effect, the local field corrections relevant to calculating $A_{\text {eff }}$ 's only apply to the free $\mathrm{OH}$ bonds directly on the surface. ${ }^{43}$ The correct selection procedure would be to select only those free $\mathrm{OH}$ bonds that 


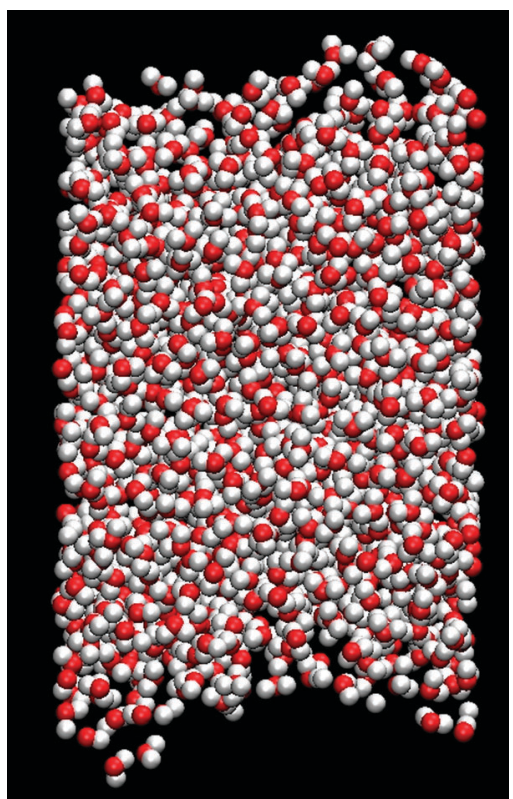

Fig. 1 Snapshot of the simulated water/vacuum interfaces. Black spheres (red, online) represent oxygen atoms, and white ones represent hydrogen atoms.

are both on the surface and those that do not contribute to bulk inversion symmetry. This aspect led us to exclude surface free $\mathrm{OH}$ bonds oriented towards the bulk and keep those oriented toward the vapor. Two experimental quantities, the surface density of free $\mathrm{OH}$ bonds, ${ }^{44} N_{s}=2.8 \times 10^{18} \mathrm{~m}^{-2}$, and their average tilt angle ${ }^{9,14,29}$ of about $35^{\circ}$, guided us in determining free surface $\mathrm{OH}$ bonds that affect the SFG results. In this study, the following three criteria were combined to select the free surface $\mathrm{OH}$ bonds which lead to agreement with the above two experimental quantities. First, an $\mathrm{OH}$ bond is considered on the surface if no oxygen atoms are inside the open cylindrical space above the hydrogen atom with a radius of 1.5 Angstrom (roughly the length of an $\mathrm{O}-\mathrm{H}$ bond). Second, the $\mathrm{OH}$ bond is not $\mathrm{H}$-bonded with any oxygen atoms. An $\mathrm{OH}$ bond is considered H-bonded with another oxygen atom if the inter-oxygen distance is less than $3.5 \AA$ (first coordination shell) and simultaneously the $\mathrm{O}-\mathrm{H} \cdots \mathrm{O}$ angle is less than $30^{\circ}$ (approximate librational wagging amplitude of the $\mathrm{H}$-bonds) ${ }^{45}$ Because of approximations in the dielectric model, some free $\mathrm{OH}$ bonds may be inappropriately included. If the bond is oriented with a large angle to the surface normal, in reality it would have a high probability to be effectively canceled out by another $\mathrm{OH}$ bond pointing in the opposite direction. Our selection method must produce an orientation ensemble that reflects this potential inversion symmetry. To address this feature, we used a single free parameter in the calculations, a free $\mathrm{OH}$ cutoff angle. Bonds with $\theta_{\mathrm{OH}}$ greater than the cutoff angle were not included in the calculations. In Table 3 the effect of choosing alternative cutoff angles is summarized. A cutoff of $60^{\circ}$ simultaneously reproduced best all experimental observables. The free surface $\mathrm{OH}$ bonds selected with the above three criteria yield the surface density of free $\mathrm{OH}$ bonds and their average angle very close to experimental values, as shown in Table 1 and Table 3, and a similar remark applies to the susceptibilities.
A snapshot of the free surface $\mathrm{OH}$ bonds is shown in Fig. 2 . Clearly, the free surface $\mathrm{OH}$ bonds in the model are sparse and their oxygen atoms do not form a uniform monolayer. The distribution of the cosine function of the tilt angle $\theta$ with respect to the surface normal is plotted in Fig. 3. As expected, the three snapshot intervals do not yield a large difference in the cosine distribution, because of the equilibrating prior to the simulation. Because a cutoff of $60^{\circ}$ was applied to the tilt angle, $\cos \theta_{\mathrm{OH}}$ cannot be smaller than 0.5, as shown in Fig. 3 . The plurality of free $\mathrm{OH}$ bonds is oriented along the surface normal.

With the surface free $\mathrm{OH}$ bonds determined, the experimental observable mode amplitudes were calculated using eqn (1)-(6). Using the damping constant $\Gamma_{q} /(2 \pi c)=14.5 \mathrm{~cm}^{-1}$ and normalizing by the simulation surface area, ${ }^{6,29}$ the data so obtained are compared with the experimental values ${ }^{29}$ and listed in Table 2. All ratios of the calculated mode amplitudes are nearly within the measured error of the experimental ratios $A_{\text {eff }}(s s p): A_{\text {eff }}(p p p): A_{\text {eff }}(s p s)=1: 0.28 \pm 0.04: 0.05 \pm 0.02$.

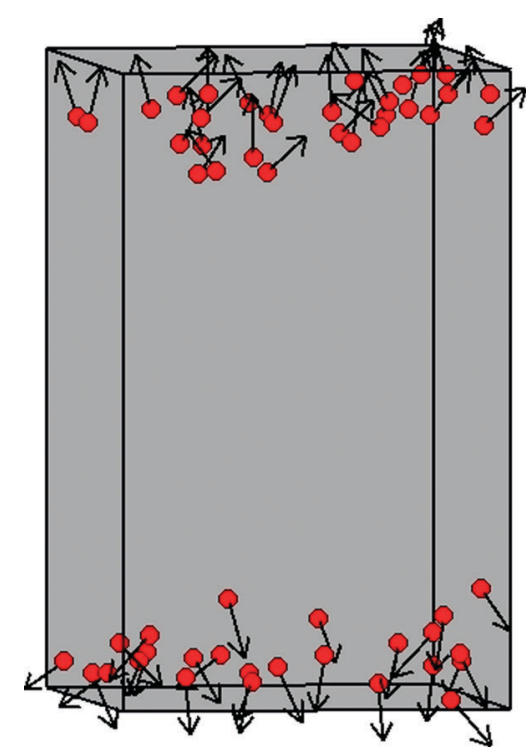

Fig. 2 Snapshot of the chosen free surface $\mathrm{OH}$ bonds. Black spheres (red, online) represent oxygen atoms and the vectors point from oxygen atoms to the bound hydrogen atoms. The vectors are elongated for clearer illustration.

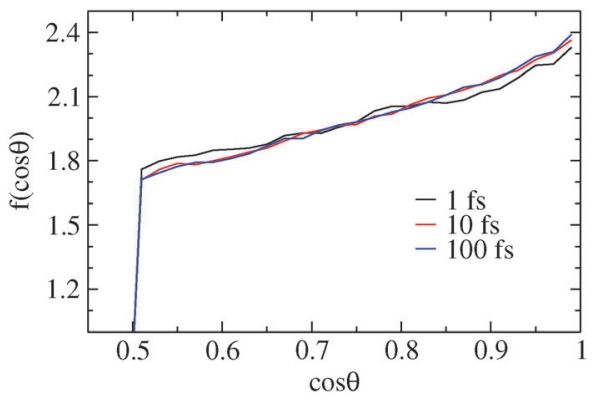

Fig. 3 Probability density of the cosine of the tilt angle with respect to the surface normal, using a cutoff angle of $60^{\circ}$. The three MD simulations with different snapshot intervals of 1,10 , and $100 \mathrm{fs}$, respectively, result in very similar cosine distributions. 
Table 2 Mode amplitudes and their ratios calculated from MD simulations and comparison with the experimental values. $A_{\text {eff }}$ has units $10^{-9} \mathrm{~m}^{2} \mathrm{~V}^{-1} \mathrm{~s}$

\begin{tabular}{lllll}
\hline Mode amplitudes & $1 \mathrm{fs}$ & $10 \mathrm{fs}$ & $100 \mathrm{fs}$ & Experimental \\
\hline$A_{\text {eff }}(s s p)$ & 0.17 & 0.35 & 3.5 & 1.7 \\
$A_{\text {eff }}(p p p)$ & 0.05 & 0.11 & 1.0 & 0.48 \\
$A_{\text {eff }}(s p s)$ & 0.002 & 0.01 & 0.3 & 0.09 \\
$A_{\text {eff }}(s s p): A_{\text {eff }}(p p p): A_{\text {eff }}(s p s)$ & $1: 0.30: 0.01$ & $1: 0.32: 0.03$ & $1: 0.30: 0.08$ & $1: 0.28 \pm 0.04: 0.05 \pm 0.02$ \\
\hline
\end{tabular}

Table 3 Simulation results depend on free $\mathrm{OH}$ cutoff angle, calculating based on the data with the snapshot interval of 1 fs. A cutoff of $60^{\circ}$ produces experimental results for a number of free $\mathrm{OH},\left\langle n_{\mathrm{OH}}\right\rangle$, average orientation angle, $\left\langle\theta_{\mathrm{OH}}\right\rangle$, and effective susceptibilities. $A_{\mathrm{eff}}$ has units $10^{-9} \mathrm{~m}^{2} \mathrm{~V}^{-1} \mathrm{~s}$

\begin{tabular}{llll}
\hline Cutoff angle & $\left\langle n_{\mathrm{OH}}\right\rangle$ & $\left\langle\theta_{\mathrm{OH}}\right\rangle$ & $A_{\text {eff }}(\operatorname{ssp}): A_{\mathrm{eff}}(p p p): A_{\mathrm{eff}}(\mathrm{sps})$ \\
\hline $45^{\circ}$ & 16 & $29^{\circ}$ & $0.07: 0.035: 0.001(1: 0.52: 0.02)$ \\
$60^{\circ}$ & 26 & $38^{\circ}$ & $0.17: 0.05: 0.002(1: 0.30: 0.01)$ \\
$90^{\circ}$ & 46 & $54^{\circ}$ & $0.40: 0.005: 0.004(1: 0.01: 0.001)$ \\
Experiment $^{29}$ & 25 & $35^{\circ}$ & $1.7: 0.48: 0.09(1: 0.28 \pm 0.04: 0.05 \pm 0.02)$ \\
\hline
\end{tabular}

The largest amplitudes in Table 3 , the $A_{\text {eff }}(s s p)$ and $A_{\text {eff }}(p p p)$, correspond to the incident IR laser being p-polarized. In p-polarization, the electric field vector is parallel to the plane of incidence and therefore probes free OH's that are nearer to the surface normal, and these free $\mathrm{OH}$ 's are those with the most orientational anisotropy. The $A_{\text {eff }}(s s p)$ is about three times larger than the $A_{\text {eff }}(p p p)$, perhaps reflecting the factor of three difference between $\beta_{\|}$and $\beta_{\perp}$. The $A_{\text {eff }}(s p s)$ is smaller than the other two, presumably because with the incident s-polarization, the IR tends to probe free OH's that are closer to being flat on the surface.

\section{Results and discussion}

The detailed information of the microscopic structure and dynamics of water/vacuum interface provided by the computer simulations provide some insight into the physical meaning of the three experimental observable mode amplitudes, the average orientation, and free $\mathrm{OH}$ bond surface density. Our results, shown in Table 2, achieve good agreement with observed ratios, and they are also within an order of magnitude of experimentally measured results. Because the $1 \mathrm{fs}$ snapshot interval led to a total integration time of $40 \mathrm{ps}$, which may be too short to adequately generate statistics (others have used multiple nanoseconds ${ }^{42}$ ), those results would expectedly differ from the longer simulation times. Although Wei and Shen obtained agreement in the calculated ratios of $A_{\text {eff }}(s s p): A_{\text {eff }}(p p p)$ by assuming the rotational dynamics of $\theta_{\mathrm{OH}}$ are very fast compared to the vibrational lifetime, they arrived at precisely $A_{\text {eff }}(s p s)=0 .{ }^{29}$ Our calculations produce a small but non-zero $A_{\text {eff }}(s p s)$, which we plan to investigate more fully comparing the slow approximation limit of the present equations. We will determine this further in future calculations. Although Gan et al. ${ }^{14}$ suggest the free $\mathrm{OH}$ bonds rarely orient perpendicularly to the surface, their results assumed the distribution of angles to be Gaussian, with a width of about 15 degrees, instead of using a distribution similar to the one calculated in Fig. 3. By calculating the distribution of the bonds and employing a cutoff, we obtain good agreement in the ratio of effective susceptibilities. We also show that not all free $\mathrm{OH}$ bonds are necessarily SFG active, and this may be a source of discrepancy between theoretical measurements of bond orientations and the narrower results from experiments. ${ }^{14}$ As expected, $A_{\text {eff }}(s s p)$ is the largest component because it represents the IR laser coupling directly to the component of the free $\mathrm{OH}$ bond oriented perpendicularly to the surface, the orientation with the largest anisotropy. Future work will reveal more information when we extend the overall results and compare them with the slow and fast approximations of the present equations.

Based on the present MD simulations of the air/water interface with the TIP3P empirical water model, the amplitudes and their ratios of the effective nonlinear susceptibilities ( $A_{\text {eff }}$ 's) were calculated for different polarization combinations and compared with sum-frequency generation (SFG) experiments. Free surface $\mathrm{OH}$ bonds are selected as the $\mathrm{OH}$ bonds (1) which are not hydrogen-bonded to other oxygen atoms; (2) whose title angles with respect to the surface normal are smaller than $60^{\circ}$; and (3) no oxygen atoms are above the hydrogen atom. With this definition of free surface $\mathrm{OH}$ bonds, the calculated average surface density and average tilt angle closely match the experimental values. The calculated absolute values of the experimental observable mode amplitudes differ, while the ratios are relatively similar. The water model presented here was utilized to capture the dynamic orientational distribution of surface free $\mathrm{OH}$ bonds. Although the present water model, TIP3P, may be satisfactory for predicting dangling surface $\mathrm{OH}$ bonds, there are indications from unpublished work that it predicts too many dangling $\mathrm{OHs}$ in bulk water, compared with experiments for non-bonded $\mathrm{OH}$ bonds. Measurements of non-bonded $\mathrm{OHs}$ in bulk water have been reported by Eaves et al. ${ }^{46}$ For studies of bulk reaction dynamics, an alternative to the TIP3P model should be explored. We are investigating how the SPC/E model, ${ }^{47}$ which has been used for bulk water structure, will alter the calculated susceptibilities. Due to the SPC/E model being better at predicting bulk water properties, we expect the enhanced correlation to bring the susceptibilities even closer to experimental results. We have not considered the effects of capillary fluctuations on the calculated effective susceptibilities, and this will be investigated in a future publication on SFG. In addition, future simulations calculating ensemble averages of 
correlation functions, instead of effective time averages, may reduce artifacts leading to variations in the calculated susceptibilities between snapshot lengths. This simulation study adds to the theoretical insight into the microscopic structure of the vacuum/water interface, and the present results provide useful information in supporting the recent theory of on-water heterogeneous catalysis. ${ }^{28}$

\section{Conclusion}

In the present paper, in summary, we have formulated the SFG equations in a form where molecular dynamics (MD) calculated quantities can be directly compared with hyperpolarizability (polarizability-dipole) coefficients, $A_{\text {eff }}$, extracted from the experimental data for different types of polarization combinations of the infrared, visible, and reflected light. Preliminary MD results were obtained and the extracted information compared with the experimental data, both in the comparison of ratios of $A_{\text {eff }}$ 's and also for comparison with their absolute values. The SFG results were interpreted in terms of physical concepts, for use in exploring other issues and with additional water models. Data from independent sources, infrared and Raman experiments, has been used, in conjunction with SFG theory, to calculate the SFG susceptibility coefficients. In this way, the overall SFG problem was divided into two parts, rather than calculating $a b$ initio the quantum mechanical values of the dipole moment and polarizability derivative. They, in practice, can be computed separately or, as above, evaluated from independent experimental data.

\section{Acknowledgements}

It is a pleasure to dedicate this article to the master of surface phenomena, friend and colleague, John Albery, on the occasion of his 75th birthday. The various authors received their support from different sources and they are pleased to acknowledge this support. $^{48}$

\section{References}

1 Y. R. Shen and V. Ostroverkhov, Chem. Rev., 2006, 106, 1140.

2 P. Jungwirth and D. J. Tobias, Chem. Rev., 2006, 106, 1259.

3 C. J. Mundy and I. F. W. Kuo, Chem. Rev., 2006, 106, 1282.

4 T. M. Chang and L. X. Dang, Chem. Rev., 2006, 106, 1305.

5 G. L. Richmond, Chem. Rev., 2002, 102, 2693.

6 Y. A. Mantz, F. M. Geiger, L. T. Molina and B. L. Trout, J. Phys. Chem. A, 2001, 105, 7037.

7 S. Narayan, J. Muldoon, M. G. Finn, V. V. Fokin, H. C. Kolb and K. B. Sharpless, Angew. Chem., Int. Ed., 2005, 44, 3275.

8 F. Niu, C.-C. Liu, Z.-M. Cui, J. Zhai, L. Jiang and W.-G. Song, Chem. Commun., 2008, 2803.

9 Q. Du, R. Superfine, E. Freysz and Y. R. Shen, Phys. Rev. Lett., 1993, 70, 2313.

10 F. Vidal and A. Tadjeddine, Rep. Prog. Phys., 2005, 68, 1095.

11 C. Y. Lee, J. A. Mccammon and P. J. Rossky, J. Chem. Phys., $1984,80,4448$.

12 K. B. Eisenthal, Chem. Rev., 1996, 96, 1343.
13 S. Gopalakrishnan, P. Jungwirth, D. J. Tobias and H. C. Allen, J. Phys. Chem. B, 2005, 109, 8861.

14 W. Gan, D. Wu, Z. Zhang, R. Feng and H. Wang, J. Chem. Phys., 2006, 124, 114705.

15 L. X. Dang and T. M. Chang, J. Chem. Phys., 1997, 106, 8149.

16 A. Morita and J. T. Hynes, Chem. Phys., 2000, 258, 371.

17 V. Buch, J. Phys. Chem. B, 2005, 109, 17771.

18 R. S. Taylor, L. X. Dang and B. C. Garrett, J. Phys. Chem., 1996, 100, 11720.

19 A. Perry, C. Neipert, B. Space and P. B. Moore, Chem. Rev., 2006, 106, 1234.

20 L. F. Scatena, M. G. Brown and G. L. Richmond, Science, 2001, 292, 908.

21 M. G. Brown, D. S. Walker, E. A. Raymond and G. L. Richmond, J. Phys. Chem. B, 2003, 107, 237.

22 I.-F. W. Kuo, C. J. Mundy, B. L. Eggimann, M. J. McGrath, J. I. Siepmann, B. Chen, J. Vieceli and D. J. Tobias, J. Phys. Chem. $B, 2006,110,3738$.

23 H.-S. Lee and M. E. Tuckerman, J. Phys. Chem. A, 2009, 113, 2144.

24 E. C. Brown, M. Mucha, P. Jungwirth and D. H. Tobias, J. Phys. Chem. B, 2005, 109, 7934.

25 A. Perry, C. Neipert, C. Ridley and B. Space, Phys. Rev. E: Stat., Nonlinear, Soft Matter Phys., 2005, 71.

26 A. Morita and J. T. Hynes, J. Phys. Chem. B, 2002, 106, 673.

27 B. M. Auer and J. L. Skinner, J. Phys. Chem. B, 2009, 113, 4125.

28 Y. Jung and R. A. Marcus, J. Am. Chem. Soc., 2007, 129, 5492.

29 X. Wei and Y. R. Shen, Phys. Rev. Lett., 2001, 86, 4799.

30 D. S. Walker, D. K. Hore and G. L. Richmond, J. Phys. Chem. B, 2006, 110, 20451.

31 X. Zhuang, P. B. Miranda, D. Kim and Y. R. Shen, Phys. Rev. B: Condens. Matter, 1999, 59, 12632.

32 X. Wei, S. C. Hong, X. W. Zhuang, T. Goto and Y. R. Shen, Phys. Rev. E: Stat. Phys., Plasmas, Fluids, Relat. Interdiscip. Top., 2000, 62, 5160.

33 Yuki Nagata and S. Mukamel, J. Am. Chem. Soc., 2010, 132, 6436.

34 P. N. Butcher and D. Cotter, The Elements of Nonlinear Optics, Cambridge University Press, 1990.

35 B. M. Auer and J. L. Skinner, J. Chem. Phys., 2008, 129, 214705.

36 Y. R. Shen, The Principles of Nonlinear Optics, J. Wiley, 2000.

37 W. Smith and T. R. Forester, The DL_POLY 2 User Manual, CCLRC, Daresbury Laboratory, Daresbury, Warrington, England, 1999.

38 W. L. Jorgensen, J. Chandrasekhar, J. D. Madura, R. W. Impey and M. L. Klein, J. Chem. Phys., 1983, 79, 926.

39 S. Nosé, Mol. Phys., 1984, 52, 255.

40 W. G. Hoover, Phys. Rev. A: At., Mol., Opt. Phys., 1985, 31, 1695.

41 J. P. Ryckaert, C. G. and H. J. C. Berendsen, J. Comput. Phys., 1977, 23, 327.

42 S. Mukamel, Principles of Nonlinear Optical Spectroscopy, Oxford University Press, 1995.

43 P. Ye and Y. R. Shen, Phys. Rev. B, 1983, 28, 4288.

44 Q. Du, E. Freysz and Y. R. Shen, Science, 1994, 264, 826.

45 A. Luzar and D. Chandler, Phys. Rev. Lett., 1996, 76, 928.

46 J. D. Eaves, J. J. Loparo, C. J. Fecko, S. T. Roberts, A. Tokmakoff and P. L. Geissler, Proc. Natl. Acad. Sci. U. S. A., 2005, 102, 13019.

47 H. J. C. Berendsen, J. R. Grigera and T. P. Straatsma, J. Phys. Chem., 1987, 91, 6269.

48 The One Hundred Talents Program of the Chinese Academy of Sciences, the Senior Visiting Scholar Program of the Chinese Academy of Sciences, the General Program of the National Natural Science Foundation of China (No. 10974208), WCU program (R31-2008-000-10055-0) through the National Research Foundation of Korea, the Biological Imaging Center of the Beckman Institute at California Institute of Technology, and also by the National Science Foundation, the Office of Naval Research, and the Army Research Office. 